\title{
Stereotactic radiosurgery for hemangiomas and ependymomas of the spinal cord
}

\author{
Stephen I. Ryu, M.D., Daniel H. Kim, M.D., and Steven D. Chang, M.D. \\ Department of Neurosurgery, Stanford University Medical Center, Stanford, California
}

\begin{abstract}
Object. The optimal treatment for intramedullary spinal tumors is controversial, because both resection and conventional radiation therapy are associated with potential morbidity. Stereotactic radiosurgery can theoretically deliver highly conformal, high-dose radiation to surgically untreatable lesions while simultaneously mitigating radiation exposure to large portions of the spinal cord. The purpose of this study was to evaluate the authors' initial experience with frameless stereotactic radiosurgery for intramedullary spinal tumors.

Methods. Between 1998 and 2003, 10 intramedullary spinal tumors were treated with stereotactic radiosurgery at the authors' institution. Seven hemangioblastomas and three ependymomas were treated in four men and three women. These patients either had recurrent tumors, had undergone several previous surgeries, had medical contraindications to surgery, or had declined open resection. Conformal treatment planning delivered a prescribed dose of 1800 to 2500 cGy (mean $2100 \mathrm{cGy}$ ) to the lesions in one to three stages. No significant treatment-related complications have been recorded. The mean radiographic and clinical follow-up duration was 12 months (range 1-24 months). One ependymoma and two hemangioblastomas were smaller on follow-up neuroimaging. The remaining tumors were stable at the time of follow-up imaging.

Conclusions. Stereotactic radiosurgery for intramedullary spinal tumors is feasible and safe in selected cases and may prove to be another therapeutic option for these challenging lesions.
\end{abstract}

\section{KEY Words • CyberKnife • spinal tumor • intramedullary lesion • stereotactic radiosurgery}

Intramedullary spinal tumors are uncommon lesions of the nervous system. Astrocytomas and ependymomas account for $90 \%$ of these, whereas the remaining tumors include hemangioblastomas, subependymomas, gangliogliomas, and metastases. ${ }^{21}$ These neoplasms may occur anywhere along the spinal cord. They commonly present with either pain or a motor or sensory deficit. Most of these tumors, with the notable exception of metastases, tend to have relatively benign clinical courses. Nevertheless, they can cause significant morbidity as they grow and affect local neural structures.

The optimal treatment for intramedullary spinal tumors is controversial. Current therapeutic options include surgery, radiation therapy, chemotherapy, or a combination of these modalities. Surgery can be safely performed in selected patients if accompanied by careful neurophysiological monitoring. Total resection results in good longterm outcomes; however, subtotal resection can be associated with tumor recurrence, especially if no adjuvant therapy is administered. ${ }^{13,21}$ Radiation therapy is an option for unresectable or residual lesions. The use of postopera-

Abbreviation used in this paper: $\mathrm{CT}=$ computerized tomography. tive radiation therapy may reduce the incidence of recurrence and improve survival in spinal cord ependymomas and astrocytomas. ${ }^{12,17}$ Chemotherapy may have a potential role in certain progressive tumors, but this role is not clearly defined as yet. ${ }^{1}$

Surgery carries the risk of paralysis. This occurs in less than $1 \%$ of patients who have no or minimal preoperative deficits, but the risk is much higher in those with more significant deficits. Most patients undergoing resection experience some postoperative deterioration, and approximately one third have significant temporary motor deficits., ${ }^{2,13,18}$ In addition, some tumors are inherently more difficult to remove. In one surgical series it was noted that thoracic spinal cord lesions and intraoperative findings of arachnoid scarring and cord atrophy were predictors of postsurgical morbidity. ${ }^{11}$ A further concern in the pediatric population is postoperative spinal deformity, which may require stabilization in up to $35 \%$ of patients. ${ }^{13}$

Radiation therapy carries the risk of radiation myelitis. This feared complication can occur after significant radiation exposure. The radiation tolerance of the spinal cord is not fully understood, which limits the amount of radiation that can be safely delivered. Some practitioners have even argued that radiation therapy should only be administered for high-grade or malignant spinal tumors. ${ }^{13}$ 


\section{S. I. Ryu, D. H. Kim, and S. D. Chang}

Given these limitations, intramedullary spinal cord lesions are suitable targets for highly conformal, high-dose radiation. Stereotactic radiosurgery permits such dose delivery while mitigating radiation injury in normal structures. In recent reports various authors have described the use of stereotactic radiosurgery for lesions of the spine, ${ }^{10,28}$ lung, ${ }^{35}$ pancreas, ${ }^{22}$ and prostate. $^{16}$ Stereotactic radiosurgery with the CyberKnife has been used to treat a variety of spinal tumors, ${ }^{8,28}$ including metastatic disease, ${ }^{23}$ benign extramedullary lesions, ${ }^{6}$ and sacral tumors. ${ }^{7,9} \mathrm{We}$ present the first report of the application of stereotactic radiosurgery to intramedullary spinal tumors. This is a retrospective review of all patients treated according to an Institutional Review Board-approved spinal stereotactic radiosurgery protocol.

\section{CLINICAL MATERIAL AND METHODS}

\section{Patient Population}

Between 1998 and 2003, seven patients with 10 intramedullary spinal cord lesions were treated with stereotactic radiosurgery at Stanford University Medical Center. Their mean age was 38 years (range 19-61 years), and there were four men and three women. Four of the five patients with hemangioblastomas had von Hippel-Lindau disease; three hemangioblastomas were treated in one patient. Two patients had ependymomas; in one patient two myxopapillary ependymomas were treated. In total, seven hemangioblastomas and three ependymomas that occurred along the spinal axis were treated with the CyberKnife frameless stereotactic radiosurgery system (Table 1).

Most patients presented with localized back pain with or without radiating down a limb. Three patients suffered a local recurrence of previously resected lesions, or new remote lesions with minimal new symptoms found on routine follow up. Two patients experienced local recurrence of previously resected lesions that had been treated with conventional radiotherapy. Two patients had local recurrence of previously resected lesions but had medical issues that prevented further surgery. Two patients felt minimally symptomatic and refused the recommended surgery. One patient had undergone multiple resections of intracranial hemangioblastomas and refused further operations on the spinal lesions.

\section{Treatment Planning and Dosimetry}

The treatment dose to the lesions was 1800 to $2500 \mathrm{cGy}$ (mean $2100 \mathrm{cGy}$ ) to the 68 to $82 \%$ isodose contour of the target volume; treatment was delivered in one to three stages. The maximum intratumor dose to the lesions ranged from 2250 to $3680 \mathrm{cGy}$ (mean $2700 \mathrm{cGy}$ ). The volume of the target lesions ranged from 0.47 to $9.8 \mathrm{~cm}^{3}$. Special care was taken to delineate the boundary of the lesion within the spinal cord to minimize exposure of the normal cord.

\section{Treatment and Follow-Up Evaluation}

The CyberKnife (Accuray, Inc., Sunnyvale, CA) is a 6-MV therapeutic linear accelerator mounted on a robotic arm that directs the treatment beam by using computer control and incorporates an x-ray imaging feedback system. During treatment, the imaging system, which consists of two orthogonally aligned x-ray cameras, acquires radiographs of targeting landmarks. The imaging guidance process analyzes these real-time radiographs and can register landmarks to the treatment planning study to determine the target's position in six dimensions. ${ }^{29}$ The process then sends control signals to the robot to adjust the originally planned treatment beam directions to accommodate for any changes in target position. This feedback obviates the need for rigid immobilization. The linear accelerator then delivers radiation beams from nearly anywhere around the patient according to the previously generated plan. This process is completely automatic and is repeated at configurable intervals during treatment. Near real-time target updating aligns the dose to the treatment volume with millimeter precision. ${ }^{24,28}$

The spinal lesion treatment protocol has been previously described. ${ }^{29}$ The current system requires implanted fiducial markers for spinal lesions; these were placed percutaneously with the aid of fluoroscopy in an outpatient

TABLE 1

Summary of characteristics and treatment parameters in seven patients with intramedullary spinal tumors*

\begin{tabular}{|c|c|c|c|c|c|c|c|c|c|c|c|}
\hline \multirow[b]{2}{*}{$\begin{array}{l}\text { Case } \\
\text { No. }\end{array}$} & \multirow[b]{2}{*}{$\begin{array}{l}\text { Age (yrs), } \\
\text { Sex }\end{array}$} & \multirow[b]{2}{*}{ Tumor Type } & \multirow[b]{2}{*}{$\begin{array}{c}\text { Prior } \\
\text { Symptoms }\end{array}$} & \multirow[b]{2}{*}{$\begin{array}{c}\text { Prior } \\
\text { Tx }\end{array}$} & \multirow[b]{2}{*}{$\begin{array}{l}\text { Tumor } \\
\text { Location }\end{array}$} & \multirow[b]{2}{*}{$\begin{array}{l}\text { RT Dose } \\
\text { (cGy) }\end{array}$} & \multirow[b]{2}{*}{$\begin{array}{l}\text { Isodose } \\
(\%)\end{array}$} & \multirow[b]{2}{*}{ Stages } & \multirow[b]{2}{*}{$\begin{array}{l}\mathrm{FU} \\
(\mathrm{mos})\end{array}$} & \multicolumn{2}{|c|}{ Outcome } \\
\hline & & & & & & & & & & Imaging & Clinical \\
\hline 1 & $41, \mathrm{~F}$ & hemangioblastoma & cervicalgia & surgery, RT & C-3 & 2250 & 77 & 2 & 24 & stable & deceased \\
\hline 2 & $24, \mathrm{M}$ & ependymoma & $\begin{array}{l}\text { LBP, LE } \\
\text { paresthesia }\end{array}$ & surgery, RT & L-3 & 1800 & 77 & 2 & 12 & decreased & improved \\
\hline 3 & $53, \mathrm{M}$ & hemangioblastoma & $\begin{array}{l}\text { cervicalgia, } \\
\text { UE paresthesia }\end{array}$ & none & $\begin{array}{c}\text { S-2 } \\
\text { C7-T1 }\end{array}$ & $\begin{array}{l}1800 \\
2100\end{array}$ & $\begin{array}{l}80 \\
78\end{array}$ & $\begin{array}{l}1 \\
3\end{array}$ & 11 & $\begin{array}{l}\text { stable } \\
\text { decreased }\end{array}$ & stable \\
\hline 4 & $61, \mathrm{M}$ & hemangioblastoma & LBP & surgery & $\mathrm{T}-12$ & 2100 & 82 & 3 & 12 & stable & stable \\
\hline 5 & $19, \mathrm{~F}$ & hemangioblastoma & cervicalgia & none & $\begin{array}{c}\text { C-3 } \\
\text { C-4 } \\
\text { C7-T1 }\end{array}$ & $\begin{array}{l}2100 \\
2100 \\
2100\end{array}$ & $\begin{array}{l}77 \\
77 \\
77\end{array}$ & $\begin{array}{l}3 \\
3 \\
3\end{array}$ & 13 & $\begin{array}{l}\text { stable } \\
\text { stable } \\
\text { decreased }\end{array}$ & stable \\
\hline 6 & $56, \mathrm{~F}$ & ependymoma & $\begin{array}{l}\text { LBP, LE } \\
\text { paresthesia }\end{array}$ & none & L1-L2 & 1800 & 70 & 1 & 1 & stable & improved \\
\hline 7 & $60, \mathrm{M}$ & hemangioblastoma & LBP & surgery & L2-L3 & 2500 & 68 & 1 & 8 & stable & stable \\
\hline
\end{tabular}

$* \mathrm{FU}=$ follow up; LBP = low-back pain; LE = lower extremity; $\mathrm{RT}=$ radiation therapy; $\mathrm{Tx}=$ treatments; UE = upper extremity. 
setting. Patients were placed on the treatment couch and a thermoplastic mask or body shell was created for gross stabilization. Patients were then moved to a Lightspeed CT scanner (General Electric Medical Systems, Milwaukee, WI), and a contrast scan was obtained. The tumor volume was outlined and inverse treatment planning was performed to achieve a highly conformal treatment plan. The generated plans minimized the dose to selected critical structures such as the spinal cord. Follow up included routine clinical visits and neuroimaging studies. The mean follow-up duration was 12 months (range 1-24 months).

\section{RESULTS}

\section{Clinical Outcomes}

All patients were treated in an outpatient setting and experienced no complications related to the treatment or fiducial placement. One ependymoma and two hemangioblastomas demonstrated radiographically confirmed decreases in size, one each at 5-, 10-, and 12-month followup review. The remaining tumors were stable at the time of last follow-up imaging. One patient with von Hippel-Lindau disease died of causes unrelated to the treatment or treated lesion. In one patient several new hemangioblastomas appeared at a distance from the treated tumor. No patient has demonstrated deterioration on neurological examination after treatment. In two patients with ependymomas, radicular symptoms improved posttreatment. In one patient, transient worsening of back pain occurred immediately after treatment. To date, no symptoms or signs of radiation myelitis have been noted.

\section{ILLUSTRATIVE CASE}

This 19-year-old woman with von Hippel-Lindau disease and multiple intracranial and spinal hemangioblastomas reported right upper- and lower-extremity sensory abnormalities; she also suffered from headaches. She was found to have multiple intracranial hemangioblastomas and three cervical spine hemangioblastomas (at C-3, C-4, and C7-T1; Fig. 1); resection was recommended. The patient was otherwise healthy and refused open surgery. The intracranial lesions were treated with CyberKnife stereotactic radiosurgery. The patient then underwent percutaneous fiducial marker placement and received CyberKnife stereotactic radiosurgery to all three spinal lesions. A prescribed dose of $2100 \mathrm{cGy}$ that was calculated to the $77 \%$ isodose line was delivered to each lesion in three stages. The treated volumes ranged from 0.36 to $0.81 \mathrm{~cm}^{3}$. The patient continues to be asymptomatic, and the C7-T1 lesion is slightly smaller at 12-month follow-up review.

\section{DISCUSSION}

Optimal resection is not always possible for intramedullary spinal tumors. This can be due to problems with accessibility, the patient's medical status, tumor characteristics, or the patient's choice. Nevertheless, symptomatic lesions should be treated. Radiation therapy has had a limited role in treatment and can potentially cause morbidity. Stereotactic radiosurgery provides for delivery of highly conformal, high-dose radiation to intramedullary lesions. In various studies, investigators have verified the dose delivery accuracy of the CyberKnife system to levels comparable with frame-based radiosurgical systems. ${ }^{3,24,28}$ We applied stereotactic radiosurgery to treat intramedullary hemangioblastomas and ependymomas.

Intramedullary hemangioblastomas account for approximately $3 \%$ of all spinal neoplasms. Isolated intramedullary hemangioblastomas are typically indolent. Complete resection results in excellent long-term functional outcome. ${ }^{19,27,33}$ These tumors should be treated when they produce symptoms or signs, because preoperative neurological function is the best predictor of long-term outcome. ${ }^{33}$ Many patients with spinal hemangioblastomas also have von Hippel-Lindau disease, a disorder associated with multiple hemangioblastomas. Although these tumors progress slowly, the high likelihood of multiple primary and recurrent lesions make the management of these hemangioblastomas more challenging. ${ }^{20,33}$ Multiple surgeries, although effective, are associated with significant discomfort and potential morbidity. Many of these tumors can be treated effectively with stereotactic radiosurgery, either alone or as an adjunct to surgery. ${ }^{26}$ For small- to moderate-sized hemangioblastomas, for multiple or recurrent tumors, and for patients who are not candidates for surgery, stereotactic radiosurgery has been shown to be a safe and effective option to control disease and improve survival, and is an attractive alternative to multiple surgical procedures. ${ }^{4,14,25}$ Results of stereotactic radiosurgery for spinal hemangioblastomas are expected to reflect the results of the same treatment for intracranial lesions.

Ependymomas, on the other hand, do not tend to be multiple but can be difficult to resect completely and often recur locally. Given their favorable long-term survival, resection is indicated for patients with intramedullary ependymomas. In one review of 33 patients, its authors noted an $86 \%$ 10-year survival rate after treatment with surgery and/or conventional radiotherapy. ${ }^{34}$ Radiation therapy is probably not necessary after total resection; however, only one fourth of tumors can be completely resected. ${ }^{5}$ Because recurrences are common, local spinal irradiation can be used after subtotal resection. Intramedullary spinal ependymomas have been noted to exhibit a radiation dose-response relationship and demonstrate a favorable long-term outcome after conventional radiation therapy. ${ }^{17}$ Theoretically, spinal stereotactic radiosurgery may be as effective as and potentially safer than conventional radiation therapy for these lesions.

Although one of the merits of stereotactic radiosurgery is its ability to focus high-dose radiation, this may not always be advantageous. Radiosurgery is a local treatment best suited for residual or nonsurgically treated tumor. Conventional wide-field radiation therapy may continue to have a role in treating tumors that are likely to recur remotely or to metastasize. Myxopapillary and high-grade cellular ependymomas are more likely than other tumors to recur remotely and may be better suited to wider field irradiation to prevent recurrence. ${ }^{34}$

Although our treatments did not lead to any immediate postprocedure complications, because intramedullary tumors are targeted directly within the spinal cord, the longterm incidence of injury is still unknown. Staging of treat- 

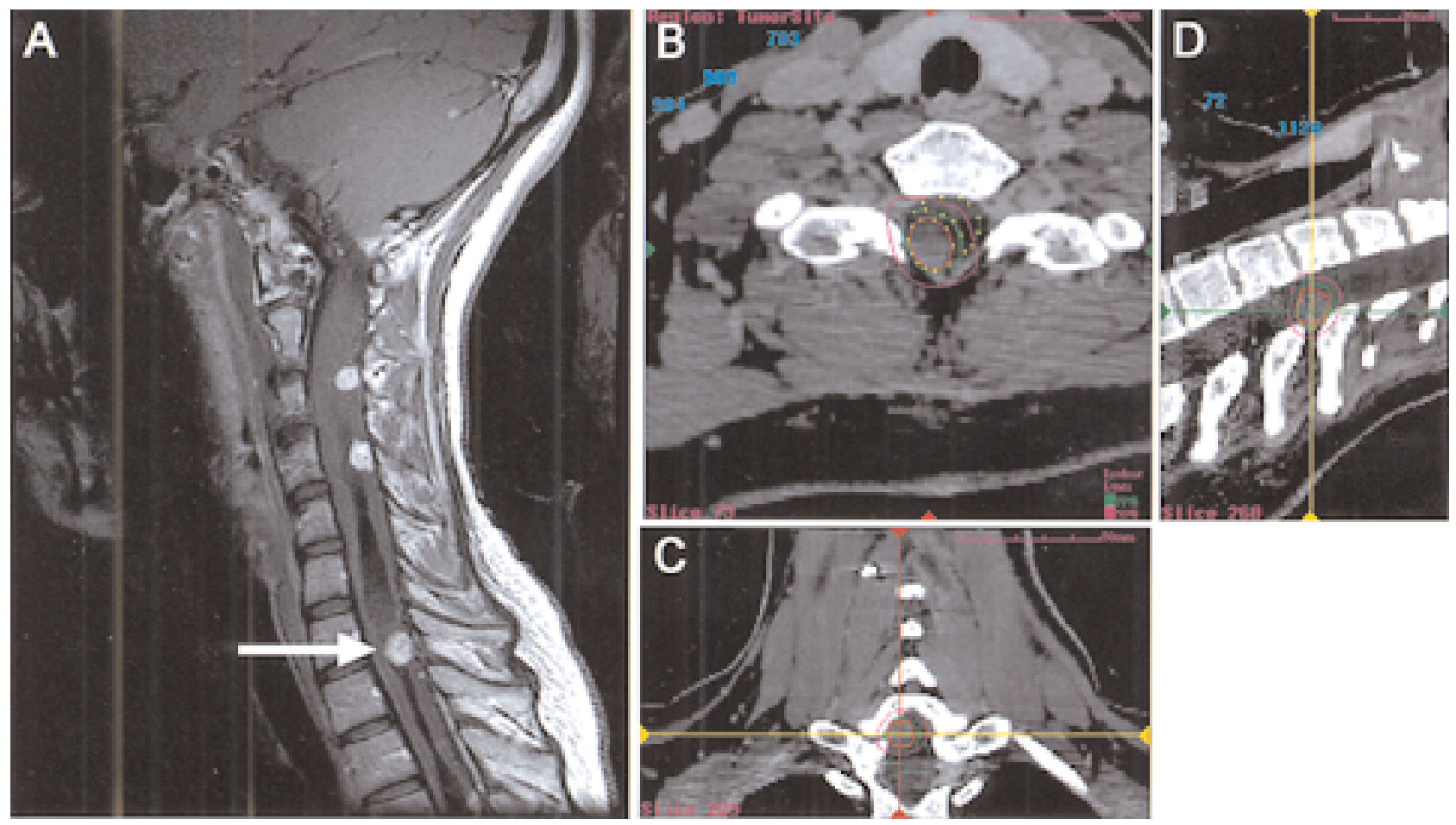

Fig. 1. Treatment plan used in a 19-year-old woman with von Hippel::Lindau disease. A: Sagittal magnetic resonance (MR) image demonstrating three hemangioblastomas at the C-3, C-4, and C7::T1 levels. There is also a large syrinx extending down to the thoracic spinal cord. B::D: Views of CT scans used in treatment planning. The patient underwent CyberKnife stereotactic radiosurgery for all three lesions; the CT-based treatment plan for the C7::T1 lesion (arrow) is presented (2100 cGy was delivered to the $77 \%$ isodose line in three 700 -cGy stages to this lesion). Axial planning MR image (B) demonstrating the targeted lesion (outlined in red), the $77 \%$ isodose line (in green), and the 50\% isodose line (in magenta). Note that the spinal cord has also been delineated as a critical structure to assist in conformal planning. Coronal (C) and sagittal (D) MR views of the same treatment plan. This lesion was smaller at 12-month follow up.

ment, precise dosimetry, and close surveillance of the patient are important factors in avoiding radiation myelitis, ${ }^{15}$ but few studies address the limits of tolerance of the spinal cord. ${ }^{30-32}$ Further study of the basic response of the spinal cord to radiation is warranted for safe guidance of future treatment.

The treatment of intramedullary spinal lesions is controversial, but many options exist. Resection remains the treatment of choice, when feasible. Stereotactic radiosurgery offers a new option for patients in whom medical causes contraindicate surgery, those who decline to undergo surgery, or those with residual or recurrent tumor after open resection.

\section{CONCLUSIONS}

Optimal treatment of intramedullary spinal tumors is a challenge. Because complete resection is not always possible, and recurrent tumor can appear after subtotal resection, and because surgery is not recommended for asymptomatic lesions, radiation therapy may have a clear role despite the risk of spinal cord injury. Stereotactic radiosurgery has been shown to be safe for a variety of spinal lesions. Our early experience supports the safety and potential benefit of stereotactic radiosurgery for primary and recurrent intramedullary spinal hemangioblastomas and ependymomas.

\section{Acknowledgment}

We thank Beth Hoyte for assistance with figure production.

\section{References}

1. Bowers DC, Weprin BE: Intramedullary spinal cord tumors. Curr Treat Options Neurol 5:207-212, 2003

2. Brotchi J, Lefranc F: Current management of spinal cord tumors. Contemp Neurosurg 21:1-7, 1999

3. Chang SD, Main W, Martin DP, et al: An analysis of the accuracy of the CyberKnife: a robotic frameless stereotactic radiosurgical system. Neurosurgery 52:140-147, 2003

4. Chang SD, Meisel JA, Hancock SL, et al: Treatment of hemangioblastomas in von Hippel-Lindau disease with linear accelerator-based radiosurgery. Neurosurgery 43:28-35, 1998

5. Clover LL, Hazuka MB, Kinzie JJ: Spinal cord ependymomas treated with surgery and radiation therapy. A review of 11 cases. Am J Clin Oncol 16:350-353, 1993

6. Gerszten PC, Ozhasoglu C, Burton SA, et al: CyberKnife frameless single-fraction stereotactic radiosurgery for benign tumors of the spine. Neurosurg Focus 14 (5): Article 16, 2003

7. Gerszten PC, Ozhasoglu C, Burton SA, et al: CyberKnife frameless single-fraction stereotactic radiosurgery for tumors of the sacrum. Neurosurg Focus 15 (2):Article 7, 2003

8. Gerszten PC, Ozhasoglu C, Burton SA, et al: Feasibility of frameless single-fraction stereotactic radiosurgery for spinal lesions. Neurosurg Focus 13 (4):Article 2, 2002

9. Gibbs IC, Chang SD: Radiosurgery and radiotherapy for sacral tumors. Neurosurg Focus 15 (2):Article 8, 2003 


\section{Radiosurgery for tumors of the spinal cord}

10. Hamilton AJ, Lulu BA, Fosmire H, et al: LINAC-based spinal stereotactic radiosurgery. Stereotact Funct Neurosurg 66: $1-9,1996$

11. Hoshimaru M, Koyama T, Hashimoto N, et al: Results of microsurgical treatment for intramedullary spinal cord ependymomas: analysis of 36 cases. Neurosurgery 44:264-269, 1999

12. Isaacson SR: Radiation therapy and the management of intramedullary spinal cord tumors. J Neurooncol 47:231-238, 2000

13. Jallo GI, Freed D, Epstein F: Intramedullary spinal cord tumors in children. Childs Nerv Syst 19:641-649, 2003

14. Jawahar A, Kondziolka D, Garces YI, et al: Stereotactic radiosurgery for hemangioblastomas of the brain. Acta Neurochir 142:641-645, 2000

15. Kim YH, Fayos JV: Radiation tolerance of the cervical spinal cord. Radiology 139:473-478, 1981

16. King CR, Lehmann J, Adler JR, et al: CyberKnife radiotherapy for localized prostate cancer: rationale and technical feasibility. Technol Cancer Res Treat 2:25-30, 2003

17. Kopelson G, Linggood RM, Kleinman GM, et al: Management of intramedullary spinal cord tumors. Radiology 135:473-479, 1980

18. Kothbauer KF, Deletis V, Epstein FJ: Motor-evoked potential monitoring for intramedullary spinal cord tumor surgery: correlation of clinical and neurophysiological data in a series of 100 consecutive procedures. Neurosurg Focus 4 (5): Article 1, 1998

19. Lee DK, Choe WJ, Chung CK, et al: Spinal cord hemangioblastoma: surgical strategy and clinical outcome. J Neurooncol 61:27-34, 2003

20. Lonser RR, Weil RJ, Wanebo JE, et al: Surgical management of spinal cord hemangioblastomas in patients with von Hippel-Lindau disease. J Neurosurg 98:106-116, 2003

21. Miller DJ, McCutcheon IE: Hemangioblastomas and other uncommon intramedullary tumors. J Neurooncol 47:253-270, 2000

22. Murphy MJ, Adler JR Jr, Bodduluri M, et al: Image-guided radiosurgery for the spine and pancreas. Comput Aided Surg 5: 278-288, 2000

23. Murphy MJ, Chang S, Gibbs I, et al: Image-guided radiosurgery in the treatment of spinal metastases. Neurosurg Focus 11 (6): Article 6, 2001
24. Murphy MJ, Cox RS: The accuracy of dose localization for an image-guided frameless radiosurgery system. Med Phys 23: 2043-2049, 1996

25. Niemela M, Lim YJ, Soderman M, et al: Gamma knife radiosurgery in 11 hemangioblastomas. J Neurosurg 85:591-596, 1996

26. Pan L, Wang EM, Wang BJ, et al: Gamma knife radiosurgery for hemangioblastomas. Stereotact Funct Neurosurg 70 (Suppl 1):179-186, 1998

27. Roonprapunt C, Silvera VM, Setton A, et al: Surgical management of isolated hemangioblastomas of the spinal cord. Neurosurgery 49:321-328, 2001

28. Ryu SI, Chang SD, Kim DH, et al: Image-guided hypo-fractionated stereotactic radiosurgery to spinal lesions. Neurosurgery 49:838-846, 2001

29. Ryu SI, Kim DH, Martin DP, et al: Image-guided spinal stereotactic radiosurgery. Techn Neurosurg 8:56-64, 2003

30. Schultheiss TE: Spinal cord radiation tolerance. Int J Radiat Oncol Biol Phys 30:735-736, 1994

31. Schultheiss TE, Kun LE, Ang KK, et al: Radiation response of the central nervous system. Int J Radiat Oncol Biol Phys 31: 1093-1112, 1995

32. Wara WM, Phillips TL, Sheline GE, et al: Radiation tolerance of the spinal cord. Cancer 35:1558-1562, 1975

33. Weil RJ, Lonser RR, DeVroom HL, et al: Surgical management of brainstem hemangioblastomas in patients with von Hippel-Lindau disease. J Neurosurg 98:95-105, 2003

34. Wen BC, Hussey DH, Hitchon PW, et al: The role of radiation therapy in the management of ependymomas of the spinal cord. Int J Radiat Oncol Biol Phys 20:781-786, 1991

35. Whyte RI, Crownover R, Murphy MJ, et al: Stereotactic radiosurgery for lung tumors: preliminary report of a phase I trial. Ann Thorac Surg 75:1097-1101, 2003

Manuscript received September 15, 2003.

Accepted in final form October 10, 2003.

Address reprint requests to: Stephen I. Ryu, M.D., Department of Neurosurgery, Stanford University Medical Center, 300 Pasteur Drive, R-297, Stanford, California 94305. email: seoulman@stanford.edu. 